\title{
Electrical potential difference and sodium and potassium fluxes across rectal mucosa in ulcerative colitis
}

\author{
C. J. EDMONDS AND DIANA PILCHER
}

From the Medical Research Council Department of Clinical Research, University College Hospital Medical School, London

SUMMARY The transmucosal electrical potential difference (pd) and the sodium and potassium flux rates (using a dialysis method) have been measured in the rectum and distal sigmoid colon of patients with ulcerative colitis and compared with measurements made in individuals having normal bowel function. In active colitis, a very low transmucosal pd was found and was associated with loss of the characteristic ability of the mucosa to absorb sodium against considerable electrochemical gradients; a marked increase in the plasma-to-lumen sodium flux rate, suggesting increased leakiness of the mucosa; and loss of the active sodium absorption mechanism. In resolving colitis, the pd was higher and all these changes of sodium transport tended to return towards normal. With full recovery, epithelial function was normal to the present tests. Potassium secretion rate showed little difference at various stages of the disease, but the nearly normal secretion of potassium in ulcerative colitis when the pd was low suggested that potassium loss to the lumen was excessive. Mucus. collected from patients with ulcerative colitis had a relatively high sodium and potassium content. Measurement of pd and absorption by using a dialysis tube offers a simple means of rapid assessment of mucosal functional integrity.

The epithelium of the colon and rectum is electrically polarized, with the luminal side normally negatively charged with respect to the blood side. Considerable changes in this electrical charge are observed during attacks of ulcerative colitis and these may persist to varying degrees as the attack resolves (Edmonds, 1970). Perfusion studies of colon during active colitis (Duthie, Watts, de Dombal, and Goligher, 1964; Harris and Shields, 1970) have shown that there is alteration in the absorption of water, sodium, and other electrolytes, but, as measurements of electrical potential difference across the epithelium were not made in these studies, the relationship of electrolyte transport to the electrical changes could not be examined. Recently a dialysis method has been applied which enables electrolyte absorption to be studied in the rectum and distal colon without perfusion (Edmonds, 1971). The method is particularly useful as by perfusion methods the transporting properties of the rectum cannot be demonstrated (Devroede and

Received for publication 3 July 1973.
Phillips, 1970). In the present report we record some: results obtained using the dialysis method to measure epithelial ionic transport in ulcerative colitis, particularly to compare the observations with the changes in potential difference. A preliminary account of the results has already been given (Edmonds and Pilcher, 1972).

\section{Methods}

\section{PATIENTS}

Measurements were made on eight patients during: and after an active exacerbation of ulcerative colitis and they were compared with measurements made on five individuals without evidence of bowel disease. No measurements were attempted when considerable bleeding was present. The most active stage examined was when the mucosa remained fragile but with little spontaneous bleeding. Consequently these patients had usually been receiving treatment with prednisone enemata but enemata were not given for at least 12 hours before the measurements. All patients were also taking: 
Salazopyrin 2 to $4 \mathrm{~g}$ by mouth daily. Suction biopsy of the rectal mucosa was done in all patients and showed varying degrees of inflammatory and other changes of ulcerative colitis, but with epithelium present.

\section{POTENTIAL DIFFERENCE}

Potential difference was measured immediately before flux measurements using a similar probe electrode to that described previously (Edmonds, 1970). The probe electrode is a lucite tube having a $\mathrm{Ag}-\mathrm{AgCl}$ junction in agar saline at its tip. It can be placed on the mucosa under direct vision by way of a sigmoidoscope and the pd at various chosen sites measured. The reference electrode system used was that described by Archampong and Edmonds (1972). It consists of a Ag-AgCl electrode in agar saline (electrically equivalent to the probe electrode) contained in a small lucite case. The reference electrode is placed on the thigh in contact with skin previously injected intradermally with physiological saline, a procedure which completely abolishes the skin pd (Archampong and Edmonds, 1972). The method allows the true transmucosal pd to be measured with a skin electrode and so is especially useful when repeated measurements are made on patients as in the present study.

\section{FLUX MEASUREMENTS}

Dialysis tubes (Visking, diameter $0.5 \mathrm{~cm}$, length $8 \mathrm{~cm}$ ), mounted on rubber catheters and containing about $1.6 \mathrm{ml}$ of solution, were placed in the rectum at chosen sites. Full details of the method with its evaluation and the calculations used to estimate flux rates have been previously described (Edmonds, 1971). Three solutions I, II, and III used in the experiments, had the following composition: I (nominal concentrations in m-equiv/1) sodium 70 , potassium 5 , choline 75 ; solution II, sodium 70 , potassium 30, choline 50; solution III, sodium 20, potassium 5, and choline 125. All solutions had a similar anion composition, chloride 120 and bicarbonate 30 . When unidirectional sodium flux rates were to be determined, a small amount of sodium-22 was added to the solutions. The amount of sodium-22 did not exceed $0.5 \mathrm{nCi}$ per tube which even if all were absorbed (for example, because of accidental leakage from the dialysis tube) would give a radiation dose of less than one thousandth of the permissible body burden of this radionuclide. Before insertion of the tube the rectum was rinsed using about $20 \mathrm{ml}$ of solution which was then completely removed by gentle suction.

The tubes were prepared immediately before insertion and weighed. They were removed after a time period, usually 30 minutes, wiped clean and reweighed and the solution was then collected from the tube. Solutions showing significant discoloration from faecal staining were discarded.

MUCUS AND EPITHELIAL SAMPLES

When sufficient mucus was seen in the rectum, samples were taken under direct vision using a PVC catheter attached to a syringe to suck up a little of the mucus. Mucus samples were delivered on to weighed aluminium foil discs, reweighed, dried in an oven at $98^{\circ} \mathrm{C}$ for 24 hours, and weighed again to determine water content. The residues were dissolved by shaking the foil discs in tubes containing $1 \mathrm{ml}$ of $0 \cdot 1 \mathrm{~N} \mathrm{HNO}_{3}$ and subsequently diluted appropriately for flame photometry.

\section{CHEMICAL AND RADIONUCLIDE}

MEASUREMENTS

Sodium and potassium were measured by flame photometry and sodium-22 in a well-type $\mathrm{NaI}(\mathrm{Th})$ crystal scintillation counter. Results are given as mean \pm 1 SEM unless otherwise stated.

\section{TERMINOLOGY}

Undirectional flux rates are indicated as $J_{L S}$ and $\mathbf{J}_{\mathrm{SL}}$ for lumen to plasma (serosal side) and plasma to lumen respectively. $J_{\text {net }}$ refers to net flux rate, absorption being positive and secretion negative. Flux rates are expressed per $\mathrm{cm}^{2}$ referring to the surface area of the dialysis tube assumed to be similar to the area of mucosal contact, and evidence has been previously presented to support this assumption in normal individuals (Edmonds, 1971). In the present investigation we have assumed that it applies similarly in patients with colitis as it seemed unlikely that the area of contact would be much different.

\section{Results}

For convenience, the results from the patients with ulcerative colitis have been divided into two groups. Group A were from patients in whom the pd of rectal mucosa was very low, being less than $10 \mathrm{mV}$ at all parts of the bowel examined. Four of these patients had had an acute attack of colitis within the four weeks before the measurements. These four had still had some symptoms, including increased frequency of bowel movement, poorly formed stools, and some bleeding. Sigmoidoscopy showed a friable mucosa. Two of the patients, who had involvement of the rectum and distal sigmoid colon only, had had an acute attack some months previously but had persistent minor symptoms and a very low potential difference. Sigmoidoscopy showed a granular, non-friable mucosa with loss of vascular 
pattern. Group B were from patients in whom the pd at all parts of the bowel examined was low or in the lower normal range but exceeded $15 \mathrm{mV}$. In these patients only minor and occasional symptoms were present. Sigmoidoscopy showed a granular non-friable mucosa with loss of vascular pattern.

\section{CONCENTRATION CHANGES}

In normal individuals, with solution I (sodium 70 m-equiv/1) in the lumen, the sodium concentration always fell considerably but with solution III (sodium 20 m-equiv/1) the change was variable in different individuals, although on average rising slightly (fig 1). In contrast, in those patients of group A having a very low pd, sodium concentration always rose with both solutions. In the patients of group B, although the changes in sodium concentration were qualitatively similar to normal, they differed significantly in degree. Thus, the fall in sodium concentration when solution $I$ was in the lumen was reduced $(P<0.05)$ while the rise in concentration with solution III was increased, though not significantly, when compared with the normals. In the case of potassium, there was little difference between the normal and colitis patients, although with solution II (potassium 30 m-equiv/1) the potassium concentration fell on average in those with acute colitis but in the normals it tended to rise.

WATER, SODIUM, AND POTASSIUM FLUXES Both in normal individuals and in those of group B the tube always lost some weight during the period of exposure indicating that water was being absorbed. There was no significant difference between the two groups, the normal loss being $0.092 \pm 0.013$ $\mu 1 \mathrm{~min}^{-1} \mathrm{~cm}^{-2}$ compared with $0.077 \pm 0.017$ $\mu 1 \mathrm{~min}^{-1} \mathrm{~cm}^{-2}$. On the other hand in those of group A only variable and small changes in weight occurred and water absorption could not certainly be demonstrated. As regards the potassium secretion rate, there appeared to be no significant difference between the normals and those with colitis(table). On the other hand, there were clear changes in sodium transport (table). These were relatively small for group $B$, the sodium absorption rate being reduced $(\mathrm{P}<0.01)$ and the plasma-to-lumen flux rate increased $(\mathrm{P} \simeq$ 0.05 ). The most striking difference observed in these experiments was, however, found when the sodium plasma-to-lumen flux rate $\left(\mathrm{J}_{\mathrm{SL}}\right)$ of the colitic patients with low pd was compared with that of the normal individuals, $J_{\mathrm{SL}}$ being more than quadrupled when the pd was very low. Since the sodium flux rate from lumen to plasma was similar in all groups, the result of the change in $\mathrm{J}_{\mathrm{SL}}$ was that the net flux altered from sodium absorption to secretion, accounting for the rise of sodium concentration, when a solution of sodium concentration of 70 m-equiv/l was placed in the lumen (fig 1).

The ratios of the observed and the expected fiux ratios were compared (table). In both the normal group and in the patients with resolving colitis, the observed flux ratios were both greater than the expected, consistent with considerable active sodium absorption by rectal mucosa (Edmonds, 1971). However, with the patients whose pd was

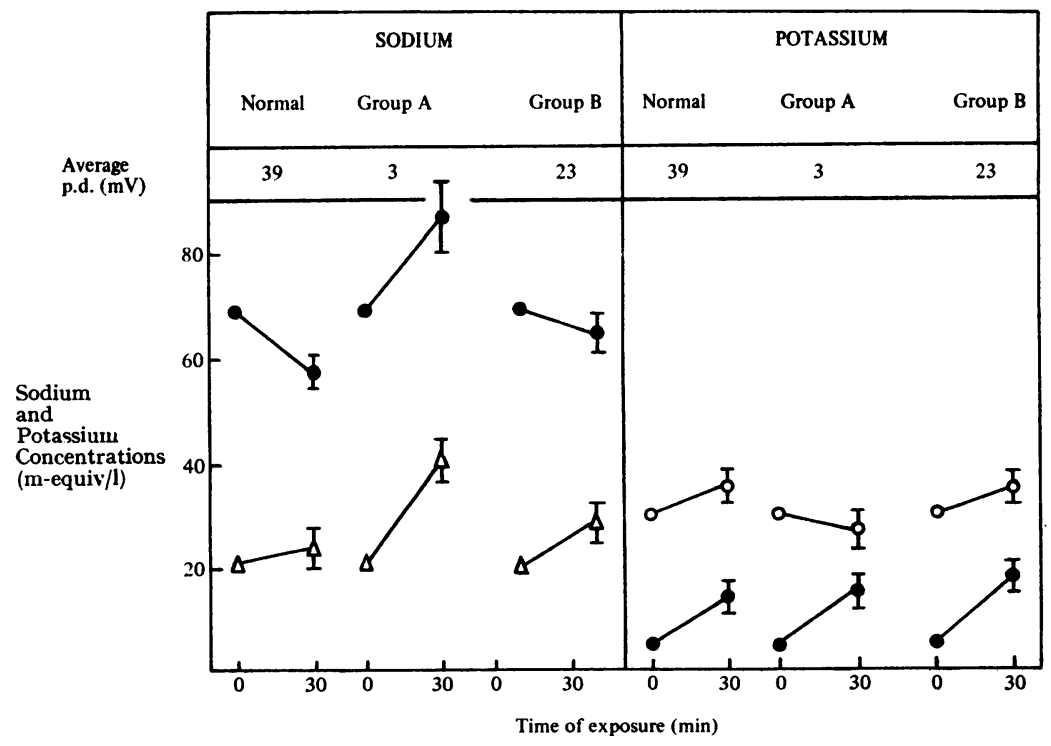

Fig 1 Changes in sodium and potassium concentration of solutions I, II, and III during 30 minutes' exposure in the rectum of five normal individuals, six of group $A$ (very low $p d)$, and seven of group $B$ (low to low-normal pd). $\odot$, solution I; $O$, solution II; $\triangle$ solution III. 


\begin{tabular}{|c|c|c|c|c|c|c|c|c|}
\hline & \multirow{3}{*}{$\begin{array}{l}\text { Number } \\
\text { of } \\
\text { Patients }\end{array}$} & \multirow{3}{*}{$\begin{array}{l}p d \\
(m V)\end{array}$} & \multicolumn{5}{|c|}{ Sodium Flux Rate ( $\mu$ Equiv min $\left.^{-1} \mathrm{~cm}^{-2}\right)$} & \multirow{3}{*}{ 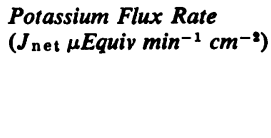 } \\
\hline & & & \multirow[t]{2}{*}{$J_{\mathbf{L S}}$} & \multirow[t]{2}{*}{$J_{\mathbf{S L}}$} & \multirow[t]{2}{*}{$J_{\text {net }}$} & \multicolumn{2}{|c|}{ Flux Ratios $\left(J_{\mathrm{LS}} / J_{\mathrm{SL}}\right)$} & \\
\hline & & & & & & Observed & Expected $^{2}$ & \\
\hline Normal & 5 & $33-45$ & $\begin{array}{r}0.095 \\
\pm 0.014\end{array}$ & $\begin{array}{r}0.044 \\
\pm 0.008\end{array}$ & $\begin{array}{l}+0.050 \\
\pm 0.010\end{array}$ & $2 \cdot 1$ & 0.09 & $\begin{array}{r}0.047 \\
\pm 0.010\end{array}$ \\
\hline Group A & 6 & $<10$ & $\begin{array}{r}0.104 \\
\pm 0.022\end{array}$ & $\begin{array}{r}0.187 \\
\pm 0.026\end{array}$ & $\begin{array}{l}-0.083 \\
\pm 0.028\end{array}$ & 0.56 & 0.46 & $\begin{array}{r}0.055 \\
\pm 0.019\end{array}$ \\
\hline Group B & 7 & $15-31$ & $\begin{array}{r}0.089 \\
\pm 0.010\end{array}$ & $\begin{array}{r}0.069 \\
\pm 0.014\end{array}$ & $\begin{array}{l}+0.019 \\
\pm 0.006\end{array}$ & $1 \cdot 3$ & $0 \cdot 14$ & $\begin{array}{r}0.061 \\
\pm 0.009\end{array}$ \\
\hline
\end{tabular}

Table Sodium and potassium flux rates measured in rectum of normal individuals and patients with colitis ${ }^{1}$

${ }^{1}$ Lumen contained solution I, sodium concentration 70 m-equiv/1, potassium concentration 5 m-equiv/1.

'Expected value based on concentration and pd, calculated from flux ratio equation (Ussing, 1949)

very low, no significant difference between the observed and expected flux ratios was found, the movements of sodium seeming here to follow the passive forces only.

The results on one patient illustrated how an apparently normally functioning epithelium could be present within a few centimetres of a completely inactive mucosa. The patient, a woman of 42 years, had had extensive colitis but part of the rectum was spared, and eight months after the present study total colectomy was carried out. The distal part of

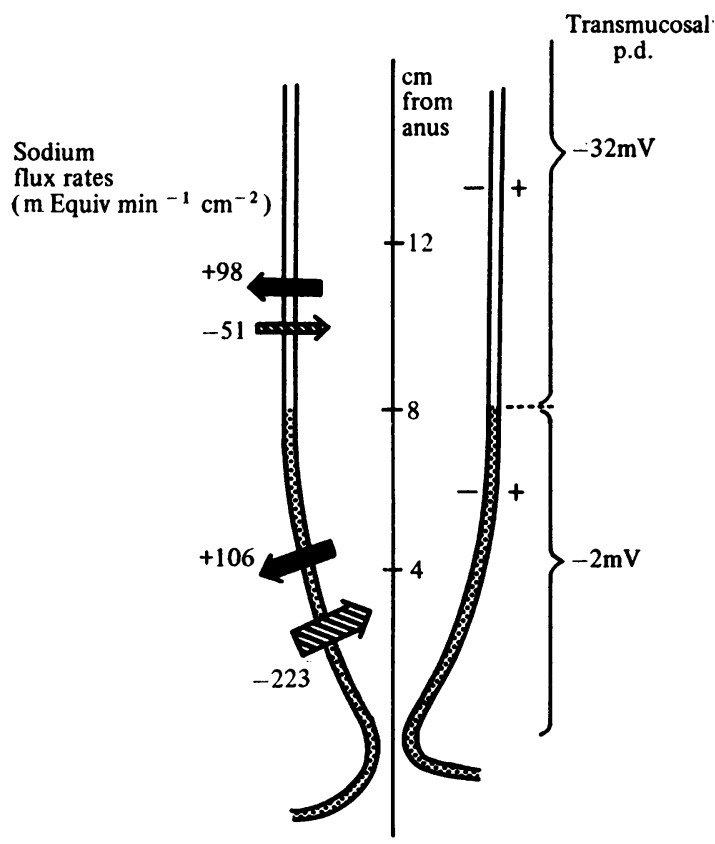

Fig 2 Potential difference and sodium flux rates measured in the rectum and distal sigmoid colon of a patient with ulcerative colitis in which part of the sigmoid colon was spared. The affected mucosa is represented diagramatically by stippling. the rectum for about 6 to $8 \mathrm{~cm}$ was diseased but the epithelium of the rest of the rectum and sigmoid colon for about $12 \mathrm{~cm}$ above appeared healthy. Potential differences were measured, and, by placing tubes at various positions within the distal gut, it was possible to examine the function of the mucosa. The results illustrated in fig 2 showed that the pd and sodium movements in the upper rectum were almost normal while in the distal and visibly affected region they were grossly abnormal. The pd change was clearly demarcated, passing from normal to practically zero over a distance of about 1 to $2 \mathrm{~cm}$.

Information about the function of the epithelium some time after apparent recovery when all treatment has been stopped is still limited. We have, however, had the opportunity to study two of the patients about two to three years after an acute attack and about one year without treatment. In both, the pd, the sodium flux rates, and potassium secretion rate were entirely within the normal range.

MUCUS

Samples obtained from the colitic patients all showed a higher sodium than potassium concentration, the mean values for sodium and potassium being $88 \pm 9$ (SD) and $42 \pm 11$ (SD) m-equiv/ $\mathrm{kg}$ net weight respectively. The water content of the mucus samples was $95 \pm 1.4 \%$ (SD).

\section{Discussion}

The normal mucosal epithelium lining the distal colon and rectum is responsible for reducing the sodium concentration in the gut lumen to a low level. This important sodium conservation function is brought about by an active sodium transport system capable of moving sodium from lumen to blood against considerable electro-chemical gradients (Edmonds, 1971). Although the quantity of sodium 
absorbed is fairly small compared with that absorbed by proximal colon, the work done per $\mu$ mole transported is very much greater because of these gradients. As appropriate to an epithelium which maintains considerable ionic gradients, the distal epithelium is relatively low in its passive permeability to sodium as reflected in the low value of the plasma-to-lumen sodium flux rate. The present study showed that several changes in these characteristic properties occurred when the epithelium was affected by the process of ulcerative colitis. First its ability to reduce the intraluminal sodium concentration to a low level was lost so that even solutions of $70 \mathrm{~m}$-equiv/ 1 sodium rose in concentration during exposure. Secondly, measurement of flux rates showed that the mucosa became 'leaky' to sodium, as indicated by the rise in the plasma-to-lumen sodium flux rate. Finally, during the active phase there appeared to be a complete loss of active sodium absorption. The fact that the flux $J_{\text {LS }}$ did not decrease may be explained by increased leakiness of the epithelium. In the normal state, $J_{L S}$ is largely due to active absorption while in active colitis it is largely due to passive diffusion. With remission, the epithelium regained its relative impermeability to sodium and its sodium transport system, although in the recovery phase some degree of impairment of sodium transport remained. It was of interest that recovery in different areas could be variable so that functionally active and inactive epithelium could exist within a few centimetres of each other.

Since four of the patients of group A were under treatment, the questions arise as to the possible effects of the prednisolone enemas on the flux measurements. To reduce the possibility of a direct modification of ionic transport processes, we omitted the enema on the evening preceding the day of study. Moreover the fact that two of the patients in group A had flux measurements essentially similar to the other members, despite having discontinued enemas several weeks previously, makes any direct effect unlikely. However, over a period of treatment prednisolone enemas may influence the ionic movements across the mucosa as the pd is observed to rise (Edmonds, 1971). This is very probably an indirect effect resulting from improvement in the mucosal state and reduction in inflammation as remission occurs.

Perfusion studies of lengths of bowel tend to be less precise as a means of analysing function of the epithelium because over long lengths epithelial function is certainly variable. On the other hand, the dialysis method is limited to the examination of the transport properties of only very short segments of gut and also is at present only employed for regions accessible from the anus. The two techniques offer to some extent complementary information, and, where comparisons are possible, the results from the two methods do show similarities. Duthie et al (1964), using bowel segments isolated at colectomy, and Harris and Shields (1970), with perfusion of the colon employing a swallowed tube, also demonstrated reduction in sodium and water absorption in active colitis and the latter also found considerably increased potassium secretion. No impairment of the ability of the epithelium to absorb sodium against considerable concentration gradients was noted, but the studies were not designed to look at this aspect.

Evidence from animal and human studies strongly suggests that the transmucosal pd of the colon is related to active sodium transport (Cooperstein and Hogben, 1959; Edmonds and Marriott, 1968; Archampong and Edmonds, 1972), although the exact mechanisms of its production are still obscure. The present study showed that with impaired sodium transport, the pd was reduced and insignificant when the epithelium had completely lost its transporting function. No reversal of pd was observed in the present study, presumably because the true transmucosal pd was measured without any contribution from skin charge which inevitably arose when perianal skin was used as the reference site as in a previous study (Edmonds, 1971). The pd is, like temperature, an intensive thermodynamic property being dependent on ionic currents and on the resistance offered by the epithelium to flow of ions (Archampong and Edmonds, 1972). Thus changes of pd can involve both aspects and the present results indicate that both do change in bowel epithelium affected by ulcerative colitis.

The present results, as well as others (Harris and Shields, 1970), suggested that potassium movement was also altered in ulcerative colitis. Normally, the pd with the lumen negatively charged would tend to facilitate the flux of potassium into the lumen and lead to the establishment of an intraluminal steady-state concentration substantially greater than that of blood. However, in active ulcerative colitis with a pd almost zero, there was still a considerable flux of potassium to the lumen and the intraluminal potassium concentration rose above the blood level. One possible explanation is that the luminal side of the membrane of the epithelial cells became abnormally permeable to potassium so that potassium ions leaked into the lumen. The cells would then tend to become depleted of potassium although continual repletion of cellular potassium from the ecf would prevent much fall in cellular potassium concentration, Of possible relevance to this hypothesis is the finding that the potassium 
content of the epithelium is reduced in ulcerative colitis (Lockwood, Harris, and Clark, 1971). However, the present data are not adequate to reach any firm conclusions. Thus the relatively high potassium secretion rate might be accounted for by enhanced mucus secretion or more rapid cell desquamation (Eastwood and Trier, 1972). Mucus obtained from the colitic patients was found to have a relatively high potassium concentration and it may well have been higher at the time of secretion since it was probably lying in the rectum for some time before its collection. Another possibility is that the low potassium of the epithelium was due to a 'sick cell' syndrome associated with impairment of the serosal side pump or it might even have resulted from depletion of mucus. Further investigations, especially to examine more directly the permeability characteristics of luminal membrane, are needed. Alterations in permeability of this barrier could affect ionic movement and also be of considerable significance in maintaining the integrity of the cells.

The present methods of measuring pd and concentrations in a dialysis tube are simple enough to be used repeatedly in an out-patient situation. They offer a means of rapidly determining the functional state of the mucosa and may be useful for clinical assessment of epithelial function. They also offer much simpler methods than colonic perfusion for study of the changes in transport characteristics of bowel epithelium produced by disease and should prove useful as research tools.

\section{References}

Archampong, E. Q., and Edmonds, C. J. (1972). Effect of luminal ions on the transepithelial electrical potential difference of human rectum. Gut, 13, 559-565.

Cooperstein, I. L., and Hogben, C. A. M. (1959). Ionic transfer across the isolated frog large intestine. J. gen. Physiol., 42, 461-473.

Devroedem, G. J., and Phillips, S. F. (1970). Failure of the human rectum to absorb electrolytes and water. Gut, 11, 438-442.

Duthie, H. L., Watts, J. M., de Dombal, F. T., and Goligher, J. C. (1964). Serum electrolytes and colonic transfer of water and electrolytes in chronic ulcerative colitis. Gastroenterology, 47, 525-530.

Eastwood, G. L., and Trier, J. S. (1972). Proliferation of the rectal epithelium in ulcerative colitis. (Abstr.) Gastroenterology, 62, 746.

Edmonds, C. J. (1970). Electrical potentials of the sigmoid colon and rectum in irritable bowel syndrome and ulcerative colitis. Gut, 11, 867-874.

Edmonds, C. J. (1971). Absorption of sodium and water by human rectum measured by a dialysis method. Gut, 12, 356-362.

Edmonds, C. J., and Marriott, J. (1968). Electrical potential and short circuit current of an in vitro preparation of rat colon mucosa. J. Physiol. (Lond), 194, 479-494.

Edmonds, C. J., and Pilcher, D. (1972). Alterations in transmucosal electrical potential difference and in sodium and potassium in the rectum produced by proctocolitis. Biol, et Gastro-ent., 5, (Arch. Mal. Appar. dig., 61, Suppl. 2), 465c.

Harris, J., and Shields, R. (1970). Absorption and secretion of water and electrolytes by the intact human colon in diffuse untreated proctocolitis. Gut, 11, 27-33.

Lockwood, C. M., Harris, J., and Clark, C. G. (1971). Intracellular potassium in diffuse proctocolitis. Lancet, 1, 889-891.

Ussing, H. H. (1949). Distinction by means of tracers between active transport and diffusion. Acta physiol. scand., 19, 43-56. 